Voix et Images

voixetimages

\title{
Entretien avec Pierre Nepveu
}

\section{Jean-François Chassay et Alexandre Drolet}

Volume 34, numéro 1 (100), automne 2008

Pierre Nepveu

URI : https://id.erudit.org/iderudit/019400ar

DOI : https://doi.org/10.7202/019400ar

Aller au sommaire du numéro

\section{Éditeur(s)}

Université du Québec à Montréal

\section{ISSN}

0318-9201 (imprimé)

1705-933X (numérique)

Découvrir la revue

Citer ce document

Chassay, J.-F. \& Drolet, A. (2008). Entretien avec Pierre Nepveu. Voix et Images, 34(1), 15-26. https://doi.org/10.7202/019400ar d'utilisation que vous pouvez consulter en ligne.

https://apropos.erudit.org/fr/usagers/politique-dutilisation/ 


\title{
ENTRETIEN AVEC PIERRE NEPVEU
}

\author{
JEAN-FRANÇOIS CHASSAY
}

Université du Québec à Montréal

\section{ALEXANDRE DROLET}

Université Laval

voIX ex IMAGES Pierre Nepveu, nous voudrions savoir d'abord comment s'est fait votre cheminement intellectuel. Le choix des études littéraires s'est-il fait rapidement, spontanément?

PIERRE NEPVEU Je dirais d'abord que mon rapport à la ville a été déterminant. J'avais très peu de contact avec la nature. Pendant longtemps, nous passions tous nos étés en ville. La famille de ma mère était de condition modeste et ma grand-mère était très religieuse. J'évoque sa mort, survenue quand j'avais 10 ans, dans Lignes aériennes. Ma mère n'avait qu'une sœur. Intellectuellement, il est sûr que j'ai été davantage marqué par la famille Nepveu, beaucoup plus nombreuse et plus instruite. La famille Nepveu, c'était d'abord mon grand-père, une figure d'autorité. Il était directeur de l'école Ollier, dans la paroisse Saint-Louis-de-France, et il était très fier d'avoir eu pour élève le futur historien Maurice Séguin, un des piliers du courant néonationaliste de l'École de Montréal. Les Nepveu n'étaient pas nécessairement des littéraires, mais ils avaient une conscience politique très marquée, ils étaient très antiduplessistes. Je suis né en 1946, j'ai donc des souvenirs de l'époque de Duplessis... Dans cette famille, la personne la plus marquante pour moi a été Estelle Nepveu, qui était une sœur de mon père un peu plus jeune que lui. Elle avait travaillé chez Fides et avait connu tout le monde dans le milieu intellectuel. Elle était une amie de Pierre Trudeau, de Simone Monet-Chartrand, d'Andrée Maillet, de bien d'autres: des gens qui avaient aussi bien évolué vers le nationalisme que vers le fédéralisme.

Il y a aussi des souvenirs plus directement littéraires. Cette tante, Estelle, lisait beaucoup. Je me souviens de la parution de Suite marine de Robert Choquette en 1953 ; je n'avais que 7 ans. C'était de la poésie qui n'avait rien à voir avec celle qui était en train de naître à l'Hexagone, mais, pour ma tante, c'était vraiment un événement littéraire. Il y a eu d'ailleurs une grande soirée poétique cette année-là à l'Hôtel Windsor, animée par Robert Choquette et à laquelle ont assisté Miron et Anne Hébert. C'est aussi l'époque où Miron rencontre Gatien Lapointe. Alors il y a ce souvenir, mais aussi celui de la bibliothèque de mon grand-père. C'était l'époque où les poèmes de Saint-Denys Garneau, puis son Journal, venaient de paraître. Je me souviens encore, j'avais 10, 11 ans, d'avoir ouvert le livre des Poésies et d'avoir trouvé bien étrange ce langage tout découpé. C'est resté latent pendant longtemps, 
mais le lien avec Saint-Denys Garneau, pour moi, est fondamental parce qu'il a ensuite été relayé par un autre événement, qui concerne cette fois mon père. En 1960 ou 1961, il reçoit en cadeau Convergences de Jean Le Moyne, et pendant plusieurs semaines ce livre traîne sur la table à café du salon.

VoIX ET IMAGES Ce n'est pas un coffee table book classique. (Rires)

PIERRE NEPVEU Non, pas vraiment! Avant ça, toujours grâce à mon père, j'avais lu Jules Verne, mais ce livre-là était d'un autre niveau intellectuel... Je l'ouvre et je tombe sur la phrase suivante: «Je ne peux pas parler de Saint-Denys Garneau sans colère», puis: "Nous avons tué Saint-Denys Garneau». Ça m’a beaucoup impressionné. On dirait que j'accède ainsi à la littérature, à la poésie. Le Moyne citait beaucoup de vers de Saint-Denys Garneau dans ce texte: "C'est eux qui m'ont tué. Ils sont tombés sur mon dos. » Je saisissais non seulement la poésie, mais aussi le côté dramatique de la littérature : le poète agressé, étouffé par son milieu. Cette lecture a été une étape déterminante. La suivante est venue plutôt de l'éducation. J'avais fait mes quatre premières années de cours classique dans le secteur public, à la Commission des écoles catholiques de Montréal (CECM), ce qui était nouveau à l'époque. J'ai donc fréquenté l'école Philippe-Aubert-De-Gaspé de 1958 à 1962 ; parmi mes confrères de classe, il y avait le futur comédien Pierre Curzi, l'éditeur Antoine Del Busso. Comme c'était une première tentative dans le public, la CECM avait recruté ses meilleurs professeurs, le programme était très exigeant, très solide. Il reste qu'à cette époque, j'étais beaucoup plus tourné vers les mathématiques que vers la littérature. J'étais fort en algèbre, en géométrie. J'adorais la géométrie. Jusqu'à l'âge de 16 ans, la poésie, la littérature n'étaient pas du tout centrales dans ma vie. Les choses ont changé à ma première année au Collège Sainte-Marie, en 1962, où j'ai entrepris la seconde moitié de mon cours classique. J'y ai alors eu un professeur, René Avon, qui a été très important dans mon itinéraire. Il nous donnait un cours de poésie moderne: les poètes du $x^{e}$ siècle, les poètes québécois également. Je me souviens d'Henri Michaux, par exemple, qui a été pour moi une découverte à l'époque. Plume a été une lecture marquante et lorsque j'ai commencé à écrire, je voulais faire du Michaux! René Avon, donc, non seulement nous a donné un enseignement moderne, mais également nourri de ce qui émergeait dans la littérature et dans la culture québécoises, en 1962-1963: les chansonniers, les apparitions de Vigneault, de Claude Léveillée...

VOIX ET IMAGES Il y a Parti pris qui arrive.

PIERRE NEPVEU Parti pris est venu un peu après, mais avec les chansonniers, c'était peut-être une conception... enfin, à travers Vigneault en tout cas, un peu plus archaïque de la poésie, avec des tonalités plus anciennes, une certaine préciosité. René Avon nous a fait découvrir tout ça. Mais le véritable déclencheur de l'écriture, ce fut sa demande d'un travail de longue haleine qui pouvait prendre n'importe quelle forme, au choix, et qui s'ajoutait aux travaux et aux examens réguliers. Je lui ai remis un petit recueil de poèmes que j'avais commencé à écrire dans un cahier. Ça lui a plu et j'ai ensuite publié dans la revue du Collège. À ce moment-là, dans le cours de René Avon, j'avais comme collègue André Brassard, qui était un personnage inoubliable...

VoIX ET IMAGES Il n'est pas resté longtemps à Sainte-Marie... 
PIERRE NEPVEU Non. Je crois qu'il n'a même pas terminé son année. On était assis côte à côte dans la classe. Je me souviens surtout de ses déclamations. Il pouvait réciter du Victor Hugo, du Baudelaire... Évidemment, c'était un peu parodique, il cabotinait beaucoup. Il avait une distance par rapport à tout ça, il ne croyait pas tout à fait à ce modèle d'éducation. Et, donc, il est parti.

Mon expérience au Collège Sainte-Marie m'a aussi fait découvrir un autre aspect de la culture, parce que le Collège se situait au coin de Bleury et de RenéLévesque (à l'époque Dorchester), donc en plein centre-ville, ce qui était nouveau pour moi. Mes amis et moi allions souvent sur la rue Crescent où il y avait à l'époque beaucoup de galeries d'art. C'est à ce moment que j'ai découvert l'art contemporain. Je me souviens de sculptures de Robert Roussil et d'Armand Vaillancourt. Il y en avait même une de Roussil qui était un échafaudage métallique, des tiges d'acier, qui a été ensuite enlevée. Un arbre aussi, sculpté par Vaillancourt. Mon intérêt pour l'art a vite pris le dessus sur les mathématiques, bien que j'aie continué à m'y intéresser sur le plan théorique. J'ai toujours gardé un grand intérêt pour les sciences, surtout les sciences physiques, les mathématiques, l'astrophysique... Par exemple, La nouvelle alliance de Prigogine et Stengers est un livre qui m'a beaucoup marqué. Cette réflexion sur la thermodynamique, sur les théories du chaos et la cybernétique est toujours restée très importante pour moi. Mais à partir de cette époque-là, mes principaux champs d'intérêt changent.

C'est aussi l'époque où on se met à lire Parti pris. Je suis arrivé au Collège à l'automne 1962, et Parti pris paraît pour la première fois en octobre 1963, et on se met alors à lire Paul Chamberland, qui publie Terre Québec en 1964, L'afficheur hurle en 1965. Après la publication de L'afficheur hurle, il vient donner une conférence au Collège Sainte-Marie. À cette époque, je dirais qu'il était pour nous LE poète qu'on lisait, avec les romanciers de Parti pris, Jacques Renaud, André Major, Laurent Girouard. L'afficheur hurle me renversait. Je me souviens d'un détail important: Chamberland avait été présenté par un professeur noir, un Antillais, qui avait fait le lien avec Aimé Césaire. Effectivement, L'afficheur hurle doit beaucoup au Cahier d'un retour au pays natal de Césaire. Je ne connaissais pas celui-ci à l'époque, mais il m'est resté quelque chose de cette filiation avec la poésie des Antilles, toute la poésie de la négritude. C'est alors que je vais choisir d'aller en lettres à l’Université de Montréal, où je continuerai à écrire.

voix ET IMAGES Comment s'est effectué ce passage?

PIERRE NEPVEU Entre cette période de quatre ans au Collège Sainte-Marie, de 1962 à 1966, et celle de trois ans à l'Université de Montréal, de 1966 à 1969, il y a une continuité sur le plan intellectuel, un élargissement, mais, sur le plan humain, j'ai vécu mon passage à l'Université de Montréal comme un exil, même si j'y ai retrouvé des confrères de collège comme Robert Melançon. Je ne me sentais pas très bien, un peu perdu dans cette immense institution. Au Collège, c'était plus convivial et j'avais des amis très proches que j'ai perdus de vue par la suite.

VoIX ET IMAGES Cela signifiait arriver dans une institution très classique...

PIERRE NEPVEU Oui, et, je dois dire, assez impersonnelle et froide, même si certains professeurs étaient très chaleureux, comme Nicole Deschamps par exemple. D'ailleurs, elle a joué un rôle important dans mon parcours. Un jour que je venais 
récupérer un travail à son bureau, elle m'a demandé ce que j'allais faire l'année suivante. Je n'avais pas le goût de me lancer tout de suite dans une maîtrise. Elle m'a dit alors qu'un de ses amis, Jack Warwick, qui avait écrit L'appel du Nord, enseignait à Hamilton et que l'Université McMaster souhaitait avoir un Québécois pour enseigner le français. Alors, je me suis dit: «Pourquoi pas?» J'ai fait ma demande et ils m'ont tout de suite convoqué en entrevue. C'est ainsi que je suis parti là-bas en septembre 1969 pour donner des cours de conversation française et aussi un cours d'introduction à la littérature québécoise. Ce fut pour moi une grande ouverture sur le monde. J'avais vécu jusqu'à 16 ans dans le quartier de la Petite-Patrie, très homogène, très canadien-français à l'époque. À Ville Saint-Laurent, où nous avions ensuite déménagé, je n'avais côtoyé aucun étranger et j'étudiais ailleurs, au centre-ville puis à l'université. Alors, à Hamilton, je me retrouve d'un seul coup avec des Français, quelques Antillais et aussi des draft-dodgers américains, des jeunes hommes comme moi qui ont fui les États-Unis... Ça m'a sensibilisé à toute la question de la guerre du Viêtnam. Pour moi ça a été une année de transition, parce qu'ensuite je suis parti étudier en France.

voIX ET IMAGES Quand vous revenez de France, en 1972, il y a déjà trois ans que vous avez publié vos premiers poèmes dans les Écrits du Canada français.

PIerRe nePveu Les textes parus dans les Écrits du Canada français, c'était en grande partie à cause de Gilles Marcotte, à qui j'avais fait lire des poèmes à l'Université de Montréal, dans un atelier de création qu'il dirigeait. Marcotte était un grand ami de Claude Hurtubise, qui dirigeait aussi HMH où j'ai ensuite publié mon premier recueil en 1971. C'est lointain et assez flou, ces premières publications: je n'habitais pas au Québec et mes débuts littéraires se sont donc produits largement in absentia. Il y a deux choses dont je me souviens quand même. Gaston Miron qui m’appelle au téléphone durant le lancement chez Hurtubise. Je n'en revenais pas! On ne se connaissait pas à l'époque et il me dit: "J'ai lu tes poèmes dans les Écrits. Continue, c'est bien que tu publies un recueil.» Miron était comme ça, il encourageait les jeunes poètes, même quand on écrivait des choses très éloignées de ce qu'il faisait. Je me rappelle aussi un article de Jean-Guy Pilon dans Le Devoir (sans doute que quelqu'un a dû me l'envoyer en France): un article assez critique qui m'a beaucoup aidé, en fait. Je pense d'ailleurs que les critiques négatives ou mitigées sont souvent plus intéressantes que les critiques positives.

voIX ET IMAGES Après le lancement de Voies rapides, vous avez repris l'avion pour la France. C'est du jet set, ça. (Rires)

PIERRE NEPVEU Un peu, oui. En même temps, mes séjours en Ontario puis en France m'ont fait rater beaucoup de choses: la Nuit de la poésie de mars 1970, la parution de L'homme rapaillé de Miron, la Crise d'octobre. Et quand je reviens au Québec au cours de l'été 1972, j'obtiens un poste à l'Université de Sherbrooke, donc un peu en retrait de la vie montréalaise.

voIX ET IMAGES Est-ce que vous avez eu l'impression, à votre retour en 1972, d'un changement dans l'atmosphère au Québec?

PIERRE NEPVEu Peut-être, oui. Les années 1960 m'avaient initié à la vie politique. En 1968, j'étais à la manifestation de la Saint-Jean au parc La Fontaine, qui a été très violente, avec des charges de la police à cheval, des bouteilles lancées de tous 
côtés... Je me souviens aussi d'être allé manifester avec les chauffeurs de taxi à l'aéroport de Dorval, une autre manifestation qui avait mal tourné, où la police avait chargé. Je dirais que c'est ce que je gardais des années 1960: contestation, révolution, même si j'étais fondamentalement un solitaire. Quand je reviens, vers 19721973, le Québec est davantage marqué par la contre-culture: c'est Mainmise, c'est un colloque sur les poètes beat, ce sont des écrivains comme Straram, Geoffroy, Francoeur, qui ont été marqués par la culture rock américaine et la culture de la drogue. Pour moi, c'est ça, les années 1970. Ensuite, sur le plan littéraire, il y a Les Herbes rouges, le courant formaliste, puis le courant féministe... Ce qui nous éloigne un peu de cette espèce de violence sociale et politique. Mais, toujours est-il que pendant ces années, comme j'enseigne à Sherbrooke, et plus tard à Ottawa, je me trouve toujours en marge, à distance. En fait, ce qui se passe de plus important pour moi, à Sherbrooke, c'est que grâce au département d'anglais voisin du département de français, je me retrouve très rapidement à North Hatley dans le groupe des écrivains anglophones. J'ai beaucoup fréquenté Ron Sutherland et le poète Doug Jones. C'est par là que je me suis retrouvé codirecteur de la revue Ellipse, avec Larry Shouldice, qui était un jeune professeur du département d'anglais. Je connaissais Ellipse depuis mes études à l'Université de Montréal. Le lien s'était fait grâce à Philip Stratford, qui enseignait alors au département d'anglais de l'Université de Montréal et qui était de la première équipe d'Ellipse avec Sheila Fischman, Doug Jones et d'autres. Stratford, qui était un ami de Gilles Marcotte, avait recruté des étudiants de notre département pour faire des traductions. J'avais donc traduit des poèmes de Frank Scott avec Charlotte Melançon. À Sherbrooke, je me suis retrouvé pendant deux ans, de 1973 à 1975, très engagé dans cette revue. Grâce à Ellipse, j'ai découvert de nombreux poètes canadiens-anglais.

VoIX ET IMAGES Sans vouloir remonter trop loin dans le temps, est-ce que c'est de là qu'est né votre intérêt pour l'Amérique, la question de l'américanité?

PIERRE NEPVEU Disons que ça a accéléré le processus. Quelques années auparavant, j'avais lu l'anthologie Contemporary American Poetry de Donald Hall, qui avait été pour moi une révélation. Il y avait des poèmes de Robert Creely, Denise Levertov et même du jeune John Ashbery. La préface de Hall allait au-delà des clichés que l'on pouvait entretenir sur la poésie américaine dans les années 1960, avec le courant beat. Il soutenait qu'il y avait deux courants principaux dans la poésie des États-Unis au $x x^{e}$ siècle : le premier était caractérisé par un travail très poussé sur la langue, une poésie assez intellectuelle, celle de Robert Lowell et de Richard Wilbur par exemple, et le second avait été nourri selon lui par Robert Frost et surtout par William Carlos Williams. Il s'agissait de retrouver l'American speech, c'est-à-dire une parole épousant le rythme de la langue parlée. En même temps, Hall parlait d'un nouveau réalisme, chez Robert Bly, Louis Simpson. Quand j'ai écrit Voies rapides, vers 19681969, je me trouvais beaucoup sous l'influence de cette anthologie, je voulais faire une poésie presque américaine, mais écrite en français.

VoIX ET IMAGES Vous parliez tout à l'heure de la contestation qui a habité les années 1960. Est-ce que c'était voulu, à travers l'aspect très urbain de ce recueil, de vous détourner de la poésie du pays de la Révolution tranquille, était-ce une forme de contestation? 
PIERRE NEPVEU Oui, nettement, et c'est un phénomène de génération. La plupart des poètes de ma génération, de différentes manières, ont ressenti le besoin de le faire, même si on avait une grande admiration pour les poètes de la génération de l'Hexagone. Les grands recueils des années 1960, aussi bien Mémoire de Jacques Brault, Pour les âmes de Paul-Marie Lapointe, que Le soleil sous la mort et Dans le sombre de Fernand Ouellette, et je pourrais en nommer plusieurs autres, avaient placé la barre très haut. Après l'espèce de point final majestueux qu'avait été L'homme rapaillé en 1970, il a fallu trouver un autre espace que celui de la poésie du pays. D'ailleurs, ce n'est pas pour rien que mon chapitre sur Miron dans Les mots à l'écoute, qui a d'abord été ma thèse de doctorat, s'appelle «Miron dépaysé ». Il s'agissait de m'éloigner encore de la perspective de la poésie du pays, pour aborder les poèmes de Miron autrement, comme écriture poétique, comme expérience dans la langue... Mais je crois que cela traduit quand même mon admiration.

VoIX ET IMAGES Trente ans après la publication de ce premier essai, vous travaillez encore sur Gaston Miron. C'est la première fois que vous abordez la biographie. Comment percevez-vous ce genre par rapport aux autres que vous avez abordés? Et que signifie une biographie sur Miron aujourd'hui?

PIERRE NEPVEU Sur le plan du travail concret, j'ai toujours été un essayiste ou un critique. Je prenais des textes et je les lisais, tout simplement. L'essentiel, c'est ce travail de lecture, qu'il s'agisse de textes consacrés ou de nouveaux recueils, comme je l'ai fait longtemps dans mes chroniques à Lettres québécoises puis dans Spirale. Dans le cas de la biographie, c'est autre chose. Le travail sur les archives, personnelles ou publiques, est tout à fait nouveau pour moi. C'est un aspect de la littérature auquel je ne m'étais même jamais intéressé. Je n'avais jamais travaillé non plus sur les brouillons d'écrivains. Cela fait aussi surgir tout un contexte, parce que, dans le fonds Miron, il y a des boîtes qui portent sur des textes politiques, des prospectus, des tracts parfois... Bien sûr, je savais que ça existait, mais ce contact matériel, c'est autre chose. Je le dois beaucoup à Marie-Andrée Beaudet. C'est elle d'abord qui m'a invité, non pas à faire la biographie - ça, c'est mon choix personnel - , mais à participer avec elle à l'édition de l'œuvre de Miron à partir des documents qu'elle possédait, une grosse malle qui se trouvait chez elle, à Québec, et qui contenait de gros dossiers, des brouillons de poèmes... Ce que je trouve fascinant là-dedans, c'est la perspective temporelle très importante, une perspective à la fois génétique et historique. Dans le travail critique, le mouvement d'écriture est préoccupé essentiellement par le travail du sens, c'est une herméneutique. Alors qu'avec la biographie, on se retrouve devant la narration. C'est un changement vraiment important, parce que raconter, c'est puiser dans un répertoire documentaire très vaste, qui déborde d'ailleurs Miron lui-même et qui touche l'histoire du Québec. Raconter, c'est aussi entrer forcément dans la chaîne des causalités. Toute séquence d'événements, quelle qu'elle soit, prend une tournure logique. On entre dans le registre des explications, des causalités, des finalités aussi, parce qu'en même temps, raconter la vie de quelqu'un dont le parcours est terminé, c'est en connaître l'aboutissement. C'est savoir, par exemple, que Miron, à partir des années 1983-1984, n'écrira pratiquement plus et c'est prendre acte de la notoriété dont il profite à cette époque. On est dans une chaîne logique qui est à la fois contraignante et stimulante. L'important, 
comme me l'a dit un jour l'écrivain et éditeur Pierre Filion, c'est de tenir la ligne du récit, parce qu'il y a quelque chose de romanesque là-dedans. Par ailleurs, il ne faut pas prétendre colmater toutes les brèches. L'enfance, la jeunesse, ça permet d'expliquer bien des choses, mais il y a le risque d'une construction trop généalogique, trop linéaire, saturée de psychologie et même de psychanalyse. Je n'aime guère les biographies qui s'embourbent dans les interprétations psychanalytiques. Il reste que je me mesure aussi à un mythe, à un personnage entré dans la légende. Un grand nombre de témoins, parents et amis, sont toujours vivants et ont leur point de vue là-dessus. Miron, de son côté, a sans cesse raconté sa vie, mais d'une façon extrêmement lacunaire, en répétant les mêmes épisodes, les mêmes événements, avec des trous immenses. Alors, forcément, mon récit est un peu polémique, il amène des remises en question, notamment sur son enfance. Tout compte fait, c'est un travail passionnant, mais d'une exigence extrême.

VoIX ET IMAGES Depuis une bonne vingtaine d'années, vous travaillez par ailleurs sur la question de l'américanité. Est-ce que c'est la littérature d'abord qui vous a attiré ou un intérêt pour l'histoire américaine?

PIERRE NEPVEU Au départ, ce n'est pas vraiment l'histoire, malgré mon intérêt pour ce sujet. C'est plus sous l'angle de lectures ponctuelles. Par exemple, quand j'étais en Ontario, je me souviens avoir lu plusieurs livres de John Updike, de Saul Bellow qui a publié Herzog à cette époque. Donc, je découvre un peu la littérature, le roman américain, ensuite, des écrivains parfois plus marginaux, Donald Barthelme, par exemple, qui écrivait des textes complètement fous. En même temps, je lis Emily Dickinson dont la poésie va toujours m'accompagner. Une opposition se dessine déjā, assez stéréotypée je le reconnais, entre Dickinson et Whitman, les deux grandes figures du XIXe siècle.

VoIX ET IMAGES Mais cette opposition est inévitable, on ne peut y échapper.

PIERRE NePVeu Sans doute... Ensuite, je me suis mis à lire sur les États-Unis, j'ai lu des revues et je me suis abonné assez rapidement à l'American Poetry Review et à la New York Review of Books. Ça m'a fait adopter un point de vue intellectuel sur la culture des États-Unis, que je développe quand même passablement dans Intérieurs du Nouveau Monde, c'est-à-dire le point de vue des intellectuels qui pensent l'histoire, qui pensent l'art et la société. Updike est un bon exemple de cette culture. Par contre, je n'ai pas été un lecteur des écrivains beat, sauf pour Ginsberg, avec Howl et Kaddish. Même Kerouac, je l'ai lu assez tardivement. Quand il est venu à Montréal en 1967 et qu'il est passé à l'émission Le sel de la semaine, je ne l'avais pas lu. À travers Emily Dickinson, j'ai également découvert le monde sédentaire, le petit monde qu'on habite, qu'on explore, la culture de la Nouvelle-Angleterre aussi. À cette époque, j'ai commencé à lire sur les puritains, sur l'installation de la culture américaine dans le Nouveau Monde. J'ai aussi lu avec passion La lettre écarlate de Hawthorne. J'y ai vu des liens certains, malgré les grandes différences, avec la culture québécoise, notamment l'importance de la religion. Je suis entré dans la réalité américaine plutôt sous cet angle-là. Je dois dire que ça a suscité en moi un certain esprit polémiste. Mais c'est une polémique douce... Pour moi, le travail critique de l'intellectuel est très important, et c'est vrai aussi par rapport au nationalisme québécois, par exemple. Dans les années 1970, il y a eu un culte de la 
génération beat, qui a fini par être omniprésent et qui m'agaçait un peu. Mais ce n'est pas une fermeture. J'ai le souvenir vif de m'être retrouvé à San Francisco en février 1984, au cœur de City Lights Books, au lancement de William Burroughs (dont je n'ai jamais été passionné), où j'ai rencontré aussi cet énergumène qu'était Ron Kovic, qui ensuite a été incarné au cinéma par Tom Cruise dans Born on the Fourth of July, titre du livre qu'avait écrit Kovic. C'est sûr que pendant ces années-là, autour de 1984, j'ai été sensibilisé à la culture américaine et davantage qu'auparavant au courant beat. San Francisco, en même temps, m'a fasciné: j'y ai retrouvé une culture américaine plurielle, et notamment l'idée de l'Amérique comme réceptacle de la culture européenne.

voix ET IMAGES Après, au Québec, notre lecture de l'Amérique s'est ouverte sur le Sud. Au début d'Intérieurs du Nouveau Monde, vous parlez de la découverte du Brésil, à l'occasion d'un colloque, qui a été pour plusieurs raisons un événement important pour vous.

PIERRE NePVEu Oui, c'est aussi le moment de l'adoption de mes filles. C'était en 1991. Ce séjour-là a duré près de quatre mois et j'ai donné deux séminaires, un à Porto Alegre, et un à São Paulo. Dès mon arrivée, j'ai participé à un grand colloque qui réunissait à la fois des Hispano-Américains, des Brésiliens, des Américains, quelques Français, et qui portait sur la question de l'Amérique. J'ai à ce moment découvert les grands écrivains brésiliens: Carlos Drummond de Andrade et tout le mouvement de la poésie brésilienne au Xxe siècle, mais aussi Guimarães Rosa, un très grand romancier. Ça a enrichi ma vision, bien que je reste pour l'essentiel un Nord-Américain.

voIX ET IMAGES C'est un peu le problème de cette question de l'américanité. Au fond, on peut bien parler des Amériques, mais quand même, les cultures au sud des ÉtatsUnis nous sont bien plus étrangères... Est-ce qu'on peut vraiment se permettre d'embrasser si large, de trouver des points communs "américains", n'y a-t-il pas un risque de noyer un peu la question?

PIERRE NEPVEU Oui, il y a ce risque de noyer des différences énormes sur l'histoire, l'histoire politique, l'histoire ethnique, l'histoire raciale, le métissage... C'est important pour la culture sud-américaine. Il reste que la question du rapport à l'Europe est fondamentalement la même dans toutes les Amériques. Mais le Sud, il ne faut pas oublier que c'est aussi la littérature haitienne, qui occupe une place importante au Québec. On est entre deux pôles inconciliables, mais il y a une circulation tout de même. Chez certains auteurs, comme Émile Ollivier, Anthony Phelps, Dany Laferrière, Joël Des Rosiers (je pense à son livre Théories caraỉbes), il y a une réflexion qui fait en sorte que ça circule, il y a des lieux de passage. Il y a un trafic, au sens du Trafic des langues de Sherry Simon, des échanges, des transactions...

voIX ET IMAGES Ce sujet nous permet de glisser vers la question de la littérature migrante. Elle rejoint un peu la question de l'américanité; vous y avez beaucoup travaillé. Qu'est-ce qu'on peut en dire aujourd'hui, après un quart de siècle de présence relative? Est-ce que c'est une littérature essentielle actuellement au Québec? Est-ce qu'elle est surévaluée, sous-évaluée? Quelle place occupe-t-elle, en fait? Est-ce que ce n'est pas très morcelé d'une certaine façon?

PIERRE NEPVEU Je voudrais rappeler que, finalement, ce problème n'est pas nouveau. Je me souviens d'avoir participé à un comité à l'UNEQ, où on avait beaucoup discuté 
de cette question. Il y avait plusieurs personnes à l'UNEQ - je pense que France Théoret, entre autres, était de celles-là - qui n'aimaient pas, et je le comprends, qu'on en fasse une catégorie. Ce comité à l'UNEQ s'occupait des manuels et des anthologies de littérature québécoise qu'on utilisait dans les écoles secondaires, les cégeps, où il y avait immanquablement, à partir des années 1985-1988...

VoIX ET IMAGES ... l'étranger de service.

PIERRE NEPVEU C'est ça, le chapitre sur «les écrivains migrants», Robin, Laferrière, etc. Ça devenait une catégorie à part. Je dois rappeler que dans L'écologie du réel, mon chapitre s'appelait «Ëcritures migrantes», mais pour moi, c'était très clair que mon intention était de saisir un imaginaire migrant qui s'actualisait, bien sûr, chez les écrivains immigrants eux-mêmes, mais qui prenait forme aussi chez des écrivains québécois d'origine, chez Hubert Aquin, Jacques Poulin, Monique LaRue, Nicole Brossard, chez plusieurs à l'époque. Cela dit, je pense que cette catégorie a été très utile. J'en parle un peu au passé, parce que je pense qu'aujourd'hui, oui, il y a des écrivains d'origine étrangère qui écrivent au Québec, mais le grand mouvement de la littérature migrante est terminé, c'est-à-dire que la réflexion s'est un peu épuisée, il faut bien le dire. D'ailleurs, j'ai participé à Rome en 2005 à un colloque sur la revue vice Versa, où se trouvaient Régine Robin et les animateurs de la revue, Lamberto Tassinari et Fulvio Caccia. On en parlait un peu comme d'un phénomène historique, c'est-à-dire quelque chose qui a essayé de se penser, de se cristalliser... Sans toujours un très grand succès d'ailleurs, car il est frappant que vice Versa ait eu une réception bien limitée et discrète dans le milieu intellectuel québécois. Les autres revues des années 1980 n’y font presque jamais référence. vice Versa était un milieu très actif, mais aussi un îlot... Cela dit, la notion d'écriture migrante a rempli le même rôle pour moi que celle de « dépaysement» dans ma lecture de Miron. Ce qui est en cause, c'est de trouver le moyen de sortir de la crispation identitaire, de penser l'identité québécoise d'une autre manière. D'ailleurs, je m'inquiète un peu de certaines dérives actuelles. Il y a des livres qui sont parus là-dessus, La dénationalisation tranquille de Mathieu Bock-Côté, par exemple, dans lesquels on se prononce contre le pluralisme, contre une vision ouverte, celle notamment de Gérard Bouchard dans La nation québécoise au futur et au passé, dont je me sens proche. Bock-Côté va jusqu'à dire que «l'appartenance nationale est inconciliable avec le pluralisme identitaire». Je m'inscris en faux contre cette affirmation, avec la plus grande véhémence.

VoIX ET IMAGES Mais c'est une formule qui semble un peu vide...

PIERRE NePVeu De son point de vue, ça semble vouloir dire quelque chose! Mais rejeter ce pluralisme ne mène nulle part. Depuis quelques années, je donne un cours à l'Université de Montréal sur les écrivains juifs au Québec, mais aussi sur le regard que les écrivains québécois, Yves Thériault à l'époque, et Myriam Beaudoin plus récemment, ont pu porter sur la culture juive. Pour moi, ce pluralisme identitaire et ce regard sur l'autre sont absolument fondamentaux. Joseph-Yvon Thériault a fait une critique de l'américanité qui était en même temps une attaque contre Gérard Bouchard, prétendant que celui-ci préconisait une liquidation du passé canadienfrançais. Je ne peux pas adhérer à cette idée, ça va à l'encontre de toutes mes convictions. Il ne s'agit pas du tout d'un reniement, d'un renoncement à ce qu'on a été, bien au contraire. Je suis un descendant des Canadiens français, il n'y a aucun doute, 
tout mon passé vient de là. Mais nous n'avons pas été que des ultramontains, des fidèles à genoux et des porteurs d'eau; et nous avons accueilli la différence, malgré une fermeture certaine de plusieurs grandes institutions, les écoles en particulier.

voIX ET IMAGES Vous avez toujours été discret sur la scène publique du côté politique, mais on a tout de même pu lire votre position sur les accommodements raisonnables à quelques reprises dans Le Devoir. Pourquoi avez-vous cru bon de prendre position cette fois?

PIERRE NePveu C'est vrai que je n'ai jamais été un militant politique comme tel, même si j'ai pu manifester... Je ne me sens pas à l'aise dans le militantisme. Pour intervenir en tant qu'intellectuel, il faut vraiment que je ressente une indignation ou une émotion forte. J'avais écrit un texte dans Le Devoir au moment du projet de loi 101. J'étais farouchement en faveur de cette loi et je le suis toujours, mais je n'aimais pas l'atmosphère dans laquelle les choses se passaient. Je me souviens, par exemple, et c'était un discours assez généralisé, qu'on disait: "Enfin, on va se comporter comme une majorité.» Ce qui signifiait quelque chose d'un peu violent: on va remettre à sa place, même faire taire cette minorité anglophone, ou alors qu'elle s'en aille! Et effectivement, il y en a plusieurs qui sont partis. Il y avait une certaine intolérance. Miron n'avait pas aimé mon texte qui était paru dans Le Devoir, il me l'avait dit, il croyait que ce n'était pas le bon moment pour publier ça.

voIX ET IMAGES C'est un peu le problème des révolutions au sens large, même si c'est sur un mode mineur au Québec. Le paradoxe d'une révolution, c'est qu'il faudrait qu'elle commence et qu'elle s'arrête tout de suite. Parce que si la révolution a lieu, c'est en réaction à quelque chose contre lequel il est généralement légitime de se battre, mais le danger est justement de réagir exactement comme l'adversaire l'a fait, et il y a sans doute eu de ça pendant un certain temps.

PIERRE NePVeu Ce qu'on peut remarquer, en tout cas, si on excepte Victor-Lévy Beaulieu et (en plus sobre!) Jacques Godbout, c'est que les écrivains québécois, en général, n'interviennent pas beaucoup. Les débats se font essentiellement entre les historiens, les sociologues, les gens de sciences politiques... Les littéraires n'ont pas beaucoup de place là-dedans.

VoIX ET IMAGES C'est vrai qu'on demande très rarement aux écrivains leur opinion sur ce qui se passe. C'est peut-être lié au statut actuel de l'écrivain au Québec.

PIERRE NEPVEU J'ai une remarque à ce propos, qui va me permettre de faire un détour par Lignes aériennes. Je pense que les écrivains québécois contemporains, en grand nombre, ont beaucoup voyagé et sont très exposés, avec une sensibilité souvent aiguë, à l'actualité internationale. Je pense notamment au très beau livre de Madeleine Gagnon, Les femmes et la guerre, qui rend compte de l'expérience de la guerre au Moyen-Orient, en Palestine... Donc, il y a une conscience mondiale. Cela dit, je trouve que les écrivains québécois, d'après ce qu'on peut observer, sont très peu branchés sur l'histoire québécoise, sur les enjeux politiques plus immédiats. Je dois dire que, pour moi, Lignes aériennes était un peu une façon de réagir à ce désinvestissement du champ politique québécois et canadien. Évidemment, je ne l'ai pas fait pour cette seule raison, mais une de mes motivations était de réagir contre une vision de l'histoire tournée vers une scène très large, mondiale, alors qu'ici même, il se passe des choses qui concernent le politique, le social et qu'on néglige. Pour moi, 
Mirabel, c'était un enjeu politique, une guerre territoriale entre le fédéral et le provincial, des expropriations et tout ce que ça comporte. Le Québec est une préoccupation très importante pour moi, même si je me suis souvent éloigné d'une perspective nationaliste. Je suis un Québécois inquiet, mais je dois dire que je suis agacé par le discours alarmiste qui nous dit constamment que le français est en régression au Québec. Discours qui joue notamment, avec perversité et mauvaise foi, sur l'ambiguïté du mot francophone, en l'utilisant dans le sens de "Canadien français», de «francophone de souche» pour nous dire, par exemple, que les francophones sont et vont devenir minoritaires à Montréal. J'ai participé l'hiver dernier à un des forums de l'Institut du Nouveau Monde. Je me trouvais dans un atelier où il y avait très peu de Québécois de souche. Il y avait quatre femmes arabes, dont deux voilées, il y avait un Latino-Américain, un Italien, un Anglo-protestant québécois. L'Anglo-protestant, qui parlait très bien français, était scandalisé par cette utilisation du mot francophone. Il disait: «On est tous des francophones ici.» Effectivement, tous parlaient un excellent français. Il y a des dérives comme ça qui nourrissent un alarmisme que je refuse de partager. Ce n'est pas que je ne suis pas inquiet, je suis très préoccupé par la minorisation croissante du Québec au Canada. En même temps, comme intellectuel, j'ai une vision tout de même positive de la culture québécoise actuelle. Je pense qu'elle est très créatrice. Ce qui fait souvent défaut actuellement dans la culture québécoise, c'est un discours de relais, qui ferait davantage le pont entre la culture savante et l'espace public. Plusieurs y travaillent, mais il faut dire aussi que l'espace public est saturé d'anti-intellectualisme...

voIX ET IMAGES Une société qui est en santé, c'est une société qui n'est pas toujours en accord avec elle-même. Un de nos problèmes est peut-être qu'on essaie toujours d'être d'accord avec nous-mêmes, y compris quand ça va mal. C'est-à-dire que quand ça va mal, tout le monde s'entend pour dire que c'est une catastrophe et quand ça va bien, on ne veut pas voir les problèmes.

PIERRE NEPVEU Oui, en effet. La recherche du consensus et même, d'une unanimité. Je pense aussi, et c'est un processus qui s'est construit sur plusieurs décennies, que la polarisation extrême au Québec dans le politique entre fédéralisme/nationalisme a fait en sorte qu'un très grand nombre de débats se sont branchés là-dessus et nous ramènent toujours là. Ça produit du silence parce que je pense que beaucoup d'écrivains québécois ne se situent pas aisément à l'intérieur de ce débat, en tout cas ressentent un malaise par rapport à celui-ci. Ou alors, ils sont eux-mêmes divisés: ils sont indépendantistes, mais leur pratique d'écrivain se situe ailleurs, sur d'autres fronts. Il y a à la fois la recherche de l'unanimité, du consensus, mais aussi la polarisation extrême, qui nous place toujours entre deux pôles, et il faut absolument prendre parti.

voIX ET IMAGES Alors que la retraite n'est plus si loin, comment percevez-vous l'importance de l'enseignement dans votre parcours intellectuel?

PIERRE NePVeu Ce que je voudrais dire là-dessus rejoint ce que je retrouve en enseignant la culture juive. Dans le judaïsme, il y a une culture de l'oralité très importante, de la parole argumentative, qui s'est développée à travers les écoles talmudiques. Dans la culture juive, la parole vive qui transmet, mais qui dialogue aussi dans la controverse, est absolument fondamentale. Il y a des textes avec des commentaires, 
mais ces commentaires sont ouverts, infinis. D'ailleurs, la déconstruction de Derrida doit beaucoup à ça. Pour moi, c'est ce que l'enseignement ouvre: un espace pour cette parole vive, dans laquelle sont relancés les textes, mais qui permet aussi d'entendre les textes. Par exemple, dans mon cours d'initiation à la poésie, on a lu collectivement «La marche à l'amour » de Miron. Au complet, pas juste trois vers, pas juste une strophe, les dix strophes. Dans un cours précédent, on avait fait «Arbres» de Paul-Marie Lapointe: c'était improvisé, les gens se relayaient un peu au hasard. Lorsque quelqu'un s'arrêtait, quelqu'un d'autre dans la salle continuait. C'est l'expérience en direct de la récitation à haute voix, de la vocalité. Évidemment, la poésie en particulier se prête beaucoup à de telles lectures. Plus généralement, l'enseignement permet aussi une circulation des voix: dans mon cours de poésie, il y a des musulmans, des Africains, des Québécois d'origine, et certains, tout à coup, prennent la parole, chacun avec son accent propre. Ça ne peut pas se vivre dans le livre, c'est un autre espace, un espace qui est physique et permet aussi à l'occasion de remettre en cause certaines valeurs, certains points de vue. Mon essai L'écologie du réel, par exemple, avait été beaucoup inspiré par le cours que je donnais dans les années 1980 sur la Révolution tranquille. On apprend parfois plus en enseignant qu'en étudiant. VoIX ET IMAGES L'enseignement aurait une dimension socratique?

PIERRE NePVeu Oui, plus que l'écrit, qui a une sorte de caractère définitif. Alors que dans l'enseignement, on retrouve une fragilité, une remise en question, voire une vulnérabilité. J'aime ce déséquilibre, ce risque, même si c'est parfois épuisant.

voIX ET IMAGES Il y a aussi une position de pouvoir qui vient avec l'enseignement, une sorte de halo...

PIERRE NEPVEu Ce prestige est aussi lié à la performance, à l'aspect théâtral de l'enseignement. Le professeur est comme un acteur sur une scène qui fait face aux spectateurs.

VoIX ET IMAGES Trois fois récipiendaire du prix du Gouverneur général, récipiendaire du prix David, membre de l'Académie des lettres, professeur à l'Université de Montréal depuis la fin des années 1970, ancien codirecteur de Spirale, tout cela fait de vous, très clairement, quelqu'un d'ancré dans l'institution, et pourtant on vous perçoit mal comme un "homme d'institution». Comment vous percevez-vous à l'intérieur de l'institution culturelle québécoise?

PIERRE NEPVEu Ce serait absurde de nier que j'appartiens à l'institution et que j'en tire un certain pouvoir. Mais par nature, j'ai toujours gardé une distance, je n'ai jamais été porté à cultiver des contacts, à déployer des stratégies. Je ne peux pas nier une reconnaissance importante, mais je suis toujours un peu étonné. Les prix du Gouverneur général ont été chaque fois une surprise pour moi. Le prix AthanaseDavid, encore bien plus! Je suis plutôt solitaire de nature. Je suis membre de l'Académie des lettres du Québec, je fais partie du comité qui organise des rencontres d'écrivains, mais ce que j'aime dans cette fonction, ce sont les rapports humains, souvent singuliers, et non les rapports institutionnels. Pour moi, la visée institutionnelle est lointaine et abstraite, même si j'en connais les enjeux. J'aime trop la littérature, la poésie, l'enseignement pour penser beaucoup à l'institution : mes énergies sont d'abord là. 